\title{
Diagnostic values of dual focus narrow band imaging and probe-based confocal laser endomicroscopy in FAP-related duodenal adenoma
}

Authors

Institutions
Rapat Pittayanon ${ }^{1}$, Rungsun Rerknimitr ${ }^{1}$, Boonlert Imraporn ${ }^{1}$, Naruemon Wisedopas ${ }^{2}$, Pinit Kullavanijaya ${ }^{1}$

${ }^{1}$ Division of Gastroenterology, Department of Medicine, Faculty of Medicine, Chulalongkorn University and King Chulalongkorn Memorial Hospital The Thai Red Cross Society, Bangkok, 10330, Thailand.

${ }^{2}$ Department of Pathology, Faculty of Medicine, Chulalongkorn University, Bangkok 10330, Thailand submitted 19. January 2015 accepted after revision 16. April 2015

\section{Bibliography}

Dol http://dx.doi.org/

10.1055/s-0034-1392235

Published online: 23.6 .2015

Endosc Int Open 2015; 03: E450-E455

(c) Georg Thieme Verlag KG Stuttgart · New York

E-ISSN 2196-9736

\section{Corresponding author}

Rapat Pittayanon, M.D

Division of Gastroenterology

Department of Internal

Medicine

Faculty of Medicine

Chulalongkorn University

Bangkok 10330

Thailand

Fax: 66-2-252-7839

rapat125@gmail.com
Background and study aims: Familial adenomatous polyposis (FAP) is associated with an increased risk of development of periampullary and nonampullary adenoma. Either routine biopsy or endoscopic removal of the lesion is generally required to identify the presence of adenoma. Because the risk of tissue sampling from the ampulla is high and nonampullary polyps are sometimes numerous, resection of all the lesions is time-consuming. This study aimed to evaluate the diagnostic values of duodenal adenoma by dual focus NBI (dNBI) and probe-based confocal endomicroscopy (pCLE) in FAP patients.

Patients and methods: The authors conducted a diagnostic study in a single tertiary-care referral center. Surveillance esophagogastroduodenoscopy with $\mathrm{dNBI}$ and pCLE was performed on 26 pa-

\section{Introduction}

$\nabla$

Familial adenomatous polyposis syndrome (FAP) is a well-known autosomal-dominant inherited disease caused by a germline mutation in the adenomatous polyposis coli (APC) tumor suppressor gene on chromosome 5q21 [1]. The estimated prevalence of this syndrome is between 1 in 5000 and 1 in 8000 [2]. Currently, all patients with FAP and their family members who carry the APC gene are scheduled for prophylactic total colectomy before the development of colon cancer [3]. Following that protocol, the mortality rate from colon cancer in these patients has been significantly decreased. Unfortunately, up to $80 \%$ of patients with FAP still develop proximal small bowel polyps $[4,5]$. In this subgroup, the incidence of duodenal cancer including ampullary cancers varies from $3 \%$ to $12 \%$ [6]. Endoscopic surveillance with end-view and side-view duodenoscopy is recommended for patients with FAP, with the interval between endoscopies varying from every 6 months to 5 years, depending on the character of the upper gastrointestinal polyps. tients with FAP for real-time adenoma diagnosis by two different endoscopists; one used dNBI and the other pCLE. Histology from the matched lesion was used as the gold standard.

Results: A total of 55 matched biopsies ( 25 ampullas, 30 nonampullas) were performed. The sensitivity, specificity, post predictive value (PPV), negative predictive value (NPV), and accuracy of dNBI vs. pCLE from all duodenal lesions were $96.9 \%$ vs. $93.8 \%, 78.3 \%$ vs. $81 \%, 86.1 \%$ vs. $88.2 \%, 94.7$ vs. $89.5 \%$, and $92.4 \%$ vs. $88.6 \%$, respectively.

Conclusions: For surveillance of periampullary and nonampullary adenoma in patients with FAP, the real-time readings provided a high degree of diagnostic value when histology was used as the gold standard. (Clinical trial registration number: NCT02162173).

Unfortunately, the technique is generally based on white light endoscopy (WLE) and the efficacy of the recommendation for endoscopy has never been confirmed [7].

In the past 10 years, many instruments including narrow band imaging (NBI) and probe-based confocal laser endomicroscopy (pCLE), have been developed to distinguish between adenoma and nonadenoma lesions in certain gastrointestinal areas, including the ampulla and surrounding area [8-10]. Unlike the previous version of the magnifying NBI endoscope, which was available only at certain endoscopic centers that focused more on research, the new dual focus NBI endoscope (dNBI) is now commercially available to community practitioners in both a 100 and 200 series. This study was designed to compare the value of using dNBI and pCLE to screen ampullary and nonampullary polyps for adenoma.
License terms

(1) $\ominus \circledast$ 

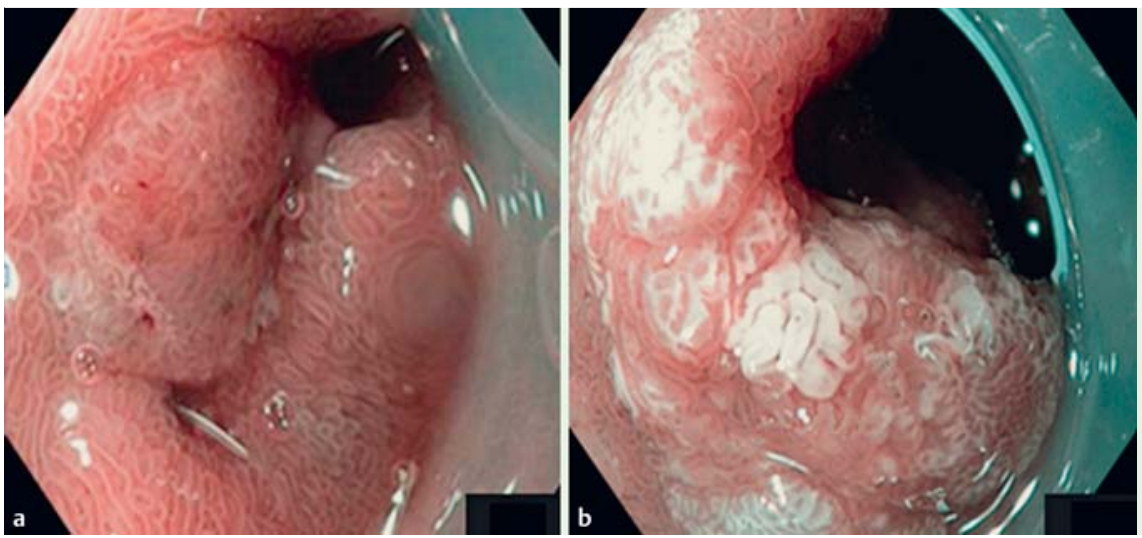

Fig. 1 The dNBI findings of 1) normal; normal or oval-shaped villi with regular vascular structures (a) and 2) adenoma; pinecone and/or leaf-shaped villi with dilated and tortuous vessels (b)
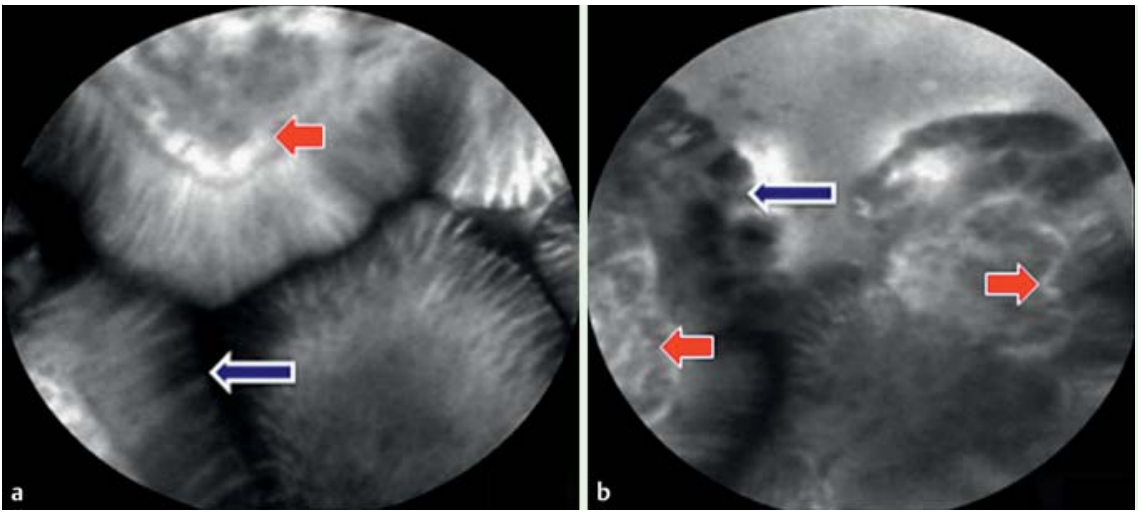

Fig. 2 The pCLE findings of 1) normal: normal epithelium border (long arrow) with regular capillary pattern (short arrow) (a) and 2) adenoma: dark/ irregular epithelium (long arrow) with tortuous capillary networks (short arrow) (b).

\section{Patients and methods}

Between December 2012 and November 2013, 26 consecutive patients previously diagnosed with FAP at the King Chulalongkorn Memorial Hospital were recruited. Male and female patients aged 18 years or older with a history of FAP and who were able to provide written informed consent were eligible. Exclusion criteria were evidence of coagulopathy (INR $\geq 1.5$ and/or platelet $<80000$, other bleeding tendency precluding biopsy, pregnancy, and allergy to fluorescein sodium. The primary outcome was definition of the diagnostic values of $\mathrm{dNBI}$ and pCLE for duodenal adenoma differentiation. The secondary outcome was exploration of new imaging findings for adenoma by dNBI and pCLE. The study protocol and consent forms were approved by the Chulalongkorn University Institutional Review Board (No. 440/55) and ClinicalTrials.gov (NCT02162173).

\section{Instrument}

An end-viewing high-definition white light endoscope (HWE) with dual focus NBI function (dNBI) (GIF-HQ 190, Olympus, Tokyo, Japan) was attached with a transparent cap (D-201-118044, Olympus, Tokyo, Japan) for detailed examination. dNBI mode was applied for real-time histology assessment. Real-time histology assessment with a confocal laser system was performed using a GastroFlex probe (pCLE, Mauna Kea Technologies, Paris, France).

\section{Endoscopic procedure and criteria for adenoma diagnosis}

Endoscopic diagnostic criteria for differentiating between adenoma and nonadenoma were adopted from the previous studies by Uchiyama Y et al. [11] The criteria for diagnosis of nonadenomatous lesions were normal or oval-shaped villi with regular vascular structures demonstrated by dNBI ( $\bullet$ Fig. 1 a) and normal epi- thelium border with regular capillary pattern demonstrated by pCLE ( Fig. 2a). The criteria for diagnosis of adenoma by dNBI was either pinecone or leaf-shaped villi (called "white villi" in this study and a term also used by another investigator [12]) with or without dilated or tortuous capillary ( Fig. $1 \mathbf{b}$ ). The criteria for diagnosis adenoma by pCLE were dark, irregular, and nonstructural mucosa with or without abnormal capillary networks ( $\bullet$ Fig. $\mathbf{2}$ b). A real-time adenoma diagnosis was made by the two independent endoscopists (BI and RP) experienced with $\mathrm{dNBI}$ and pCLE readings. Prior to participating in the study, they had experience in reviewing images obtained with $\mathrm{dNBI}$ and pCLE and had published related results elsewhere [13-15].

During the study period, all 26 patients underwent the procedure under conscious sedation with intravenous midazolam and meperidine. Before the procedure, $10 \mathrm{mg}$ of hyoscine was administered to decrease intestinal peristalsis. At the beginning of the procedure, the first endoscopist (BI) used an end-viewing high-definition white light endoscope attached to an endoscopic cap to examine the first and second part of the duodenum. The cap was used to facilitate a proper enface view of the ampulla and surrounding area ( $\bullet$ Fig. 1 a). All applicable ampullary and nonampullary (duodenal) polyps larger than $1 \mathrm{~mm}$ were examined in this study. The dNBI mode then was switched on for real-time diagnosis by the first endoscopist (BI). Subsequently, the second endoscopist (RP), who was blinded to the NBI reading, was called from another room to perform pCLE examination. During pCLE evaluatiom, $2.5 \mathrm{~mL}$ of $10 \%$ fluorescein (Novartis Pharmaceutical Corporation, Bangkok, Thailand) was injected. In the case of multiple nonampullary lesions, the first endoscopist only examined the lesions with pCLE but did not inform the second endoscopist about the result of the dNBI readings. Polypectomy was then performed by the second endoscopist. The duration 
Patient and disease characteristics $(\mathrm{N}=\mathbf{2 7})$

Age (mean $\pm S D$ ), years

Gender, male

Age of FAP diagnosis (mean \pm SD), (max,min), years

History of colectomy

Age at colectomy (mean \pm SD), (max, min) years

Colon cancer

Age at colon cancer diagnosis (mean \pm SD), (max,min) years

Family history of FAP

Number (\%)
$38.9 \pm 12.2$
$6(22.2)$
$31.8 \pm 13.0,(10,56)$
$26(96.3)$
$32.5 \pm 12.7,(15,56)$
$7(25.9)$
$38.3 \pm 11.1(24,51)$
$18(66.7)$

Number (\%)

$6(22.2)$

$31.8 \pm 13.0,(10,56)$

$26(96.3)$

7 (25.9)

$18(66.7)$
Table 1 Patient, disease, and

procedure characteristics of the entire procedure and all complications related to the procedures were recorded.

\section{Histological assessment}

All polypectomy specimens were immersed in formalin and sent for histological examination. The specimens were stained with hematoxylin and eosin (H\&E) and reviewed by an experienced gastrointestinal pathologist (NW) blinded to the endoscopic diagnosis. Definitive diagnosis was based on the Vienna classification[16] for differentiation between adenoma and non-adenoma.

\section{Interobserver agreement}

Two endoscopists (BI and RP) interpreted all of the off-line dNBI and pLE images from this study to evaluate for interobserver agreement.

\section{Statistical Analysis}

By using histology as the gold standard, the diagnostic values of dNBI and pCLE for ampullary and non-ampullary polyp interpretation were assessed for sensitivity, specificity, positive predictive value (PPV), negative predictive value (NPV), and accuracy. For numerical variables, the results were expressed as a mean \pm $\mathrm{SD}$, whereas other quantitative variables were expressed as percentages. Cohen's kappa ( $\mathrm{K}$ ) was used to analyze interobserver agreement. The value of kappa ( $\mathrm{K}$ ) for agreement was graded as slight agreement for 0.01 to 0.20 , fair for 0.21 to 0.40 , moderate for 0.41 to 0.60 , substantial for 0.61 to 0.80 , and almost perfect for 0.81 to 1.00.SPSS version 17.0 (SPSS (Thailand) Co., Ltd., Bangkok, Thailand) for Windows was used for statistical analysis.

\section{Results}

$\nabla$

A total of 57 images were obtained from 27 patients. The mean age of patients with FAP was $38.9 \pm 12.2$ years and most were female $(77.8 \%)$. The average age at diagnosis of FAP was $31.8 \pm 13.0$ years (10-56). Of the patients, 26 of 27 (96.3\%) underwent colectomy at a mean age of 32.4 years and seven (25.9\%) already harbored adenocarcinoma in the surgical specimen. One 19- year-old female has been scheduled for a prophylactic colectomy in a year. Eighteen of 27 (66.7\%) had a family history of FAP ( $\triangle \mathrm{Ta}$ ble 1 ).

One patient was excluded from the study on the day of examination because she refused to have a biopsy, although she underwent the full spectrum of endoscopic examination. Another two refused fluorescein injection and they were recruited only for the dNBI part. Ultimately, there were 55 matched biopsies ( $25 \mathrm{am}$ pullas and 30 nonampullas) with $55 \mathrm{dNBI}$ readings and 53 pCLE readings. Completion of a dNBI examination required $17.0 \pm 7.6$ $\mathrm{min}$, whereas PCLE required $12.3 \pm 6.7 \mathrm{~min}$. The mean size of ampullary and nonampullary polyps was $2.6 \pm 3.3 \mathrm{~mm}$ and $2.2 \pm 4.5$ $\mathrm{mm}$, respectively. Of those 55 lesions, the number of ampullary and nonampullary adenoma diagnosed with dNBI, pCLE, and histology were $36(65 \%), 34(64 \%)$, and $32(58 \%)$, respectively. For the primary outcome, the overall sensitivity, specificity, positive predictive value (PPV), negative predictive value (NPV), and accuracy of dNBI vs. pCLE were $96.9 \%$ vs. $93.8 \%, 78.3 \%$ vs. $81 \%, 86.1 \%$ vs. $88.2 \%, 94.7$ vs. $89.5 \%$, and $92.4 \%$ vs. $88.6 \%$, respectively, using histology as the gold standard ( $\bullet$ Table 2 ).

Both dNBI and PCLE were 100\% accurate for all stages of the Spigelman classification, with the exception of stage IV because no patients enrolled had a stage IV polyp ( $\bullet$ Table 3 ).

Approximately $80 \%$ of lesions with adenoma (27 of 32 ) met the criteria for whit villi. In addition, we found a new endoscopic feature for adenoma diagnosis in one patient who did not have white villi. In that individual, pCLE revealed dark, irregular, variable thickening of epithelial borders, which we termed "bumpy." The "bumpy" criterion was $100 \%$ accurate for diagnosis of adenoma in all five lesions with those characteristics.

The interobserver agreement for dNBI readings was rated as substantial (Kappa 0.78) and that for pCLE was almost perfect (Kappa 0.95).

Table 2 Diagnostic values of dual focus narrow band imaging and probe-based confocal laser endomicroscopy in FAP-related duodenal adenoma

\begin{tabular}{|lllllll|}
\hline Location with method (N) & Sensitivity (\%) & Specificity (\%) & PPV (\%) & NPV (\%) & Accuracy (\%) & False negative (\%) \\
\hline Ampulla with dNBI (25) & 91.7 & 92.3 & 91.7 & 92.3 & 92 & 8.3 \\
\hline Ampulla with pCLE (23) & 91.7 & 90.9 & 91.7 & 90.9 & 91.3 & 8.3 \\
\hline Non-ampullary polyp with dNBI (30) & 100 & 60 & 83.3 & 100 & 92.8 & 0 \\
\hline Non-ampullary polyp with pCLE (30) & 95 & 70 & 86.3 & 87.5 & 86.6 & 5 \\
\hline Both locations with dNBI (55) & 96.9 & 78.3 & 86.1 & 94.7 & 92.4 & 3.1 \\
\hline Both locations with pCLE (53) & 93.8 & 81 & 88.2 & 89.5 & 88.6 & 6.2 \\
\hline
\end{tabular}

dNBI, dual focus narrow band imaging; pCLE, probe-based confocal laser endomicroscopy.

1 In two cases, pCLE was not used because of lack of patient consent. 
Table 3 Accuracy of dNBI and pCLE according to Spigelman classification

\begin{tabular}{|llll|}
\hline & Number of patients (\%) & Accuracy by dNBI & Accuracy by pCLE \\
\hline Spigelman stage 0 & $9(35 \%)$ & $100 \%$ & $100 \%$ \\
\hline Spigelman stage I & $5(19 \%)$ & $100 \%$ & $100 \%$ \\
\hline Spigelman stage II & $6(23 \%)$ & $100 \%$ & $100 \%$ \\
\hline Spigelman stage III & $6(23 \%)$ & $100 \%$ & $100 \%$ \\
\hline Spigelman stage IV & 0 & NA & NA \\
\hline
\end{tabular}

$\mathrm{dNBI}$, dual focus narrow band imaging; $\mathrm{PCLE}$, probe-based confocal laser endomicroscopy; NA, no data.
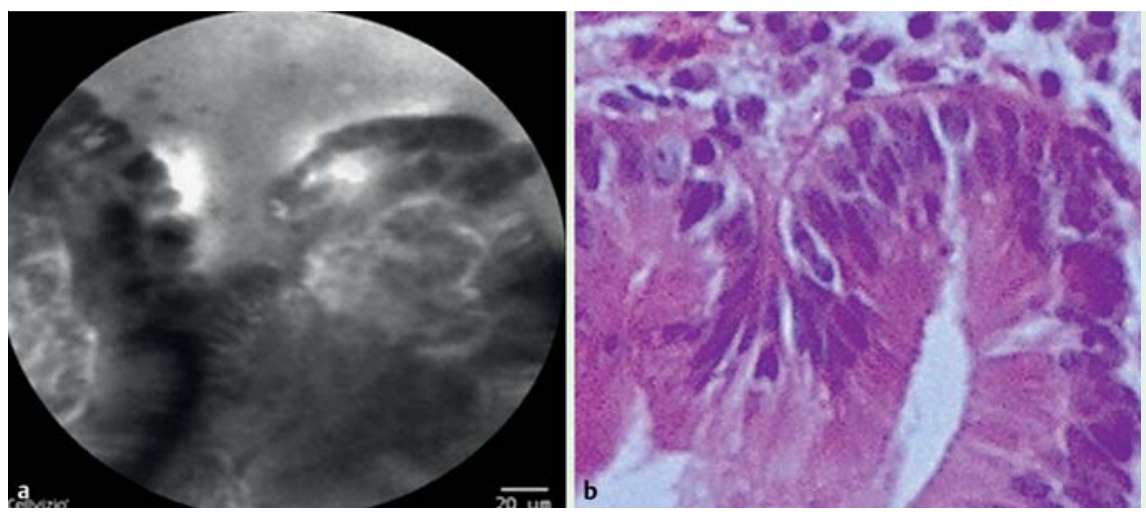

Fig. 3 The corresponding adenoma finding from PCLE and pathological image

\section{Discussion}

$\nabla$

Conventional WLE may be optimal for duodenal polyp detection but it is considered suboptimal for characterization [17]. In the current series, 23/55 (42\%) polyps were confirmed by histology as nonadenoma, making it a potentially useful technique for characterizing lesions. Risk associated with removal of duodenal polyps is significant [18] and removal of nonadenomatous polyps is definitely unnecessary. In addition, performance of random biopsies has been shown to have varying accuracy for adenoma diagnosis (40-85\%) [19-22]. Conventional chromoendoscopy also has been attempted to improve the yield for duodenal polyp detection $[17,23,24]$. Kiesslich $R$, et al. reported improved outcome with indigocarmine chromoendoscopy than with WLE (98 vs. 28 lesions; $P=0.0042$ ) for detection of duodenal abnormality in 118 patients [23]. Unfortunately, the dye spray technique is cumbersome and only helpful for screening a suspicious lesion, and targeted biopsy is still required to confirm histology.

In recent years, digital chromoendoscopy including NBI has been introduced for duodenal polyp evaluation. In 2006, based on experience in 14 patients with FAP who underwent surveillance upper gastrointestinal endoscopy, Uchiyama Y et al. [11] published the endoscopic patterns of ampullary polyps under NBI, which were classified as I, oval-shaped villi; II, pinecone/leafshaped villi (called "white villi" in this study); or III, irregular/ nonstructured villi. An excellent correlation (100\%) was demonstrated between patterns II and III and the histology of adenoma and adenocarcinoma. However, the three criteria have yet to be validated in any subsequent study. Using different criteria, Shahid et al. adopted the dysplastic Barrett features read by NBI and pCLE as their means of diagnosing duodenal adenoma [25]. In their study, they compared the efficacy of non-magnifying NBI and PCLE for diagnosis of adenoma in 65 gastric and duodenal polyps [25]. They showed a trend toward superior efficacy in diagnosis of gastric and duodenal adenoma for pCLE compared with non-magnifying NBI $(P=0.8)$, (sensitivity $92 \%$ vs. $83 \%$, specificity $78 \%$ vs. $78 \%$, PPV $71 \%$ vs. $69 \%$, NPV $94 \%$ vs. $89 \%$ and accu- racy $83 \%$ vs. $80 \%$ ) [24] With the improvement in image quality plus dual focus function of the new NBI, the current study demonstrated much higher efficacy for dNBI in diagnosis of duodenal adenoma compare to conventional NBI. However, the efficacy of our pCLE readings was good and in keeping with those reported in the previous study [25]. In addition, the current series contained only duodenal adenoma (not other lesions).

In our experience, because of enhancement of the vessels in the lamina propria (seen as deep blue color) with dNBI, we observed better delineation of both villi and vascular patterns. Moreover, the white villi with well-demarcated borders were better appreciated with dNBI. Apart from the better quality of the new NBI, we speculate that the higher efficacy of our NBI readings may be related to the more specific criteria for duodenal adenoma that we used [12]. On the other hand, the pCLE images of adenoma that showed dark, irregular, and nonstructural mucosa and sometimes abnormal capillary networks corresponded well to the histology of adenoma, which typically contains elongated, stratified columnar cells with increased nuclear-cytoplasmic ratio, known as "atypical columnar cells" ( $\bullet$ Fig. 3 ).

In the current series, dNBI and pCLE demonstrated lower specificity for adenoma in nonampullary than in ampullary lesions (65\% vs. $91 \%$ ). We found that the pattern of white villi could also be seen in non-neoplastic duodenal polyps, whereas the ampulla hardly contains non-neoplastic white villi. The white villi are believed to be lipid droplets which have substantially accumulated within the enterocytes [26]. No supporting data yet exist to explain the mechanism of lipid accumulation in duodenal adenoma or how to differentiate them from non-neoplastic mucosa. However, we observed that the majority of neoplastic white villi appeared denser than non-neoplastic white villi ( $\bullet$ Fig. 4). Unfortunately, because all patterns of white villi were defined as adenoma in this study, false-positive readings may occur in some non-neoplastic lesions with white villi. On the other hand, a false-negative reading in a small duodenal adenoma also may occur if the pCLE probe (diameter $2.3 \mathrm{~mm}$ ) is misplaced in nearby 

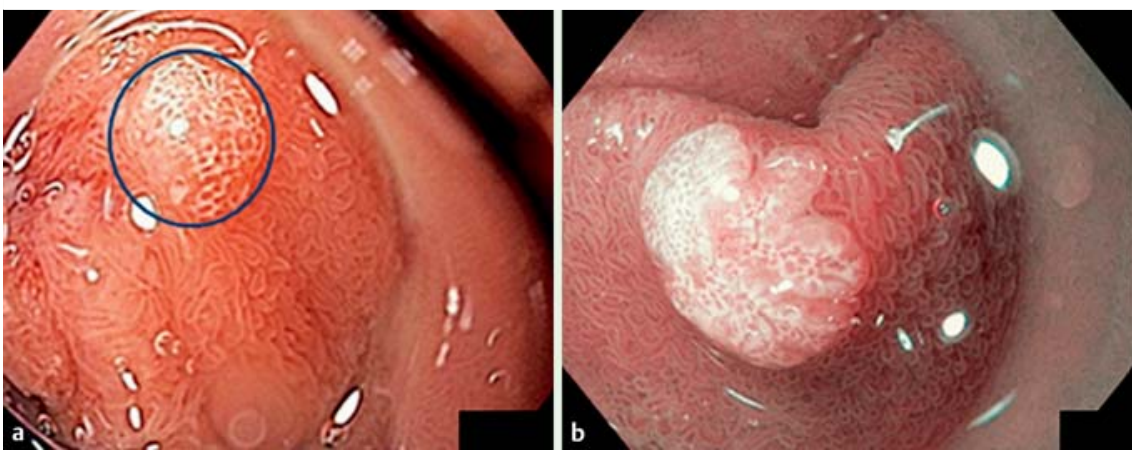

Fig. 4 a partial rim of white villi under white light represented non-adenoma (blue circle), $\mathbf{b}$ dense white villi under dNBI represented adenoma.

normal mucosa. In our experience, false-negative diagnosis by pCLE sometimes occurs in polyps smaller than $2.5 \mathrm{~mm}$.

Spigelman classification [7] can sort the risk of duodenal adenoma development and has been used to guide the frequency of upper endoscopic surveillance in FAP patients. This classification takes into consideration the number and size of polyps, and polyp histology [5]. Unfortunately, in many studies, the scheduled surveillance protocol according to the Spigelman classification has been shown not to prevent development of unresectable ampullary cancer $[27,28]$. A modified Spigelman classification, which omits moderate dysplasia from pathologic score, recently has been introduced, with the aim of reducing the difficulty of pathology reading but it yet to be validated. It also does not include ampullary adenoma, which is associated with the highest risk of development of cancer. Moreover, polyp histology is still required for staging. In the current, study without the need for polyp histology, we found perfect (100\%) concordance between real-time histological readings (either dNBI or pCLE) and the standard Spigelman classification (only in stage 0-III) ( $\bullet$ Table 3 ). Hypothetically, pCLE may be able to distinguish between the histologies of tubular adenoma (TA), tubulovillous adenoma (TVA), and villous adenoma (VA). Unfortunately, all specimens in the current study were confirmed by pathology as TA without TVA or VA and graded as Spigelman stage 0 - III without stage IV by pCLE. The results can only be used to read Spigelman stage 0 to III polyps containing TA and it did not address the importance of histological differentiation between TA, TVA, and VA by pCLE. Therefore, before we can introduce this into standard practice, we need to validate this protocol in a larger FAP population that includes Spigelman stage IV and TVA/VA.

The high NPV in both diagnosis and characterization of the lesion, however, supports the excellent efficacy of a new instrument [29]. The NPV of dNBI was more than $90 \%$ for both ampullary and nonampullary adenoma interpretation whereas the NPV for pCLE was more than $90 \%$ for only ampullary adenoma interpretation. Although the NPV for CLE in nonampullary adenoma readings did not reach $90 \%$, it was $88 \%$. In addition, the PPVs of dNBI and pCLE were high and acceptable for both ampullary and nonampullary adenomas (83\%-92\%) ( Table 2). The adenoma detectedin the current study was indeed an adenoma and not a lesion of "lesser pathology," such as Brunner's gland hyperplasia or a lesion of benign duodenal histology. Thus unnecessary endoscopic resection was not an issue. Moreover, we demonstrated a low rate of false negatives across all categories of lesions ( $0 \%-8 \%)$ ( Table 2). To translate this protocol into practice, we may use either dNBI or pCLE for the diagnosis of both ampulla and nonampullary duodenal polyps. That practice may save time both on examination during upper gastrointestinal surveillance and perhaps on the cost of polyp specimen assessment if dNBI is used.
We also identified a "bumpy lesion" as another representative finding of nonampullary adenoma. This lesion has been mentioned in a recent Japanese review article as nodular protrusion [30]. It can be easily identified under either HWE or dNBI, whereas on pCLE, findings for a bumpy lesion were similar to those for non-bumpy adenoma. Although a bumpy characteristic was found in only one patient, that patient had 5 such lesions, all of which were confirmed as adenoma by histology.

The main limitation of our study is the number of patients with FAP who presented for an upper gastrointestinal endoscopy surveillance. For this series, we were able to recruit only 27 patients with FAP and was no high-grade dysplasia was documented. Therefore, we were unable to demonstrate the benefit of dNBI and PCLE in grading of dysplasia. Moreover, the number of patients was not sufficient to perform a randomized controlled trial for a direct comparison between dNBI and pCLE. However, instead of using patient numbers we used the number of duodenal polyps and ampulla as the representative population in this study $(n=55)$, which helped us to calculate the statistical significance of the sensitivity, specificity, PPV, NPV, and accuracy between the two different methods. Another limitation is that the skills on pCLE and dNBI readings of the two endoscopists have never been compared directly with a crossover design. However, all photos and video clips from these two modalities were recorded and internally audited by the two endoscopists. The interobserver agreements in dNBI and pCLE interpretation between them were excellent (kappa 0.78-0.95). Moreover, this study definitively lacks a third arm with white light recognition of adenoma versus nonadenomatous lesions. A further study should include white light mode in the protocol to demonstrate the efficacy of WLE for lesion detection and characterization. Finally, in this study, an end-viewing endoscope was used instead of a sideviewing scope because the dNBI function was not available on the side-viewing model during the study period.

\section{Conclusions \\ $\nabla$}

In conclusion, dNBI and pCLE are both useful for real-time adenoma reading in patients with FAP who present for upper gastrointestinal endoscopy surveillance. Both methods achieved NPVs of more than $90 \%$. Practically, dNBI is a more reasonable method, considering the high cost of pCLE. Real-time histology may replace standard histology in patients with FAP, especially those with Spigelman stage 0 - III lesions.

\section{Competing interests: None}




\section{References}

1 Groden J, Thliveris A, Samowitz W et al. Identification and characterization of the familial adenomatous polyposis coli gene. Cell 1991; 66: 589-600

2 Rustgi AK. Hereditary gastrointestinal polyposis and nonpolyposis syndromes. N Engl J Med 1994; 331: 1694-1702

3 Lynch HT, de la Chapelle A. Hereditary colorectal cancer. N Engl J Med 2003; 348: 919-932

4 Kadmon M, Tandara A, Herfarth C. Duodenal adenomatosis in familial adenomatous polyposis coli. A review of the literature and results from the Heidelberg Polyposis Register. Int J Colorectal Dis 2001; 16: $63-75$

5 Spigelman $A D$, Williams $C B$, Talbot IC et al. Upper gastrointestinal cancer in patients with familial adenomatous polyposis. Lancet 1989; 2 : $783-785$

6 Jagelman DG, DeCosse JJ, Bussey HJ. Upper gastrointestinal cancer in familial adenomatous polyposis. Lancet 1988; 1: 1149-1151

7 Burke CA, Beck GJ, Church JM et al. The natural history of untreated duodenal and ampullary adenomas in patients with familial adenomatous polyposis followed in an endoscopic surveillance program. Gastrointest Endosc 1999; 49: 358 - 364

8 Dekker E, Fockens P. Advances in colonic imaging: new endoscopic imaging methods. Eur J Gastroenterol Hepatol 2005; 17: 803-808

9 Kuiper T, van den Broek FJ, van Eeden S et al. Feasibility and accuracy of confocal endomicroscopy in comparison with narrow band imaging and chromoendoscopy for the differentiation of colorectal lesions. Am J Gastroenterol 2012; 107: 543-550

10 Shahid MW, Buchner AM, Heckman MG et al. Diagnostic accuracy of probe-based confocal laser endomicroscopy and narrow band imaging for small colorectal polyps: a feasibility study. Am J Gastroenterol 2012; 107: $231-239$

11 Uchiyama $Y$, Imazu $H$, Kakutani $H$ et al. New approach to diagnosing ampullary tumors by magnifying endoscopy combined with a narrow band imaging system. J Gastroenterol 2006; 41: 483-490

12 Lopez-Ceron M, van den Broek FJ, Mathus-Vliegen EM et al. The role of high-resolution endoscopy and narrow band imaging in the evaluation of upper GI neoplasia in familial adenomatous polyposis. Gastrointest Endosc 2013; 77: 542 - 550

13 Pittayanon R, Rerknimitr R, Wisedopas $N$ et al. Flexible spectral imaging color enhancement plus probe-based confocal laser endomicroscopy for gastric intestinal metaplasia detection. J Gastroenterol Hepatol 2013; 28: $1004-1009$

14 Pittayanon $R$, Rerknimitr $R$, Wisedopas $N$ et al. The learning curve of gastric intestinal metaplasia interpretation on the images obtained by probe-based confocal laser endomicroscopy. Diagn Ther Endosc 2012; 2012: 278045

15 Rerknimitr $R$, Imraporn $B$, Klaikeaw $N$ et al. Non-sequential narrow band imaging for targeted biopsy and monitoring of gastric intestinal metaplasia. World J Gastroenterol 2011; 17: 1336-1342
16 Schlemper RJ, Riddell RH, Kato $Y$ et al. The Vienna classification of gastrointestinal epithelial neoplasia. Gut 2000; 47: 251 - 255

17 Picasso M, Filiberti R, Blanchi $S$ et al. The role of chromoendoscopy in the surveillance of the duodenum of patients with familial adenomatous polyposis. Dig Dis Sci 2007; 52: 1906-1909

18 Norton ID, Gostout CJ. Management of periampullary adenoma. Digestive diseases 1998; 16: $266-273$

19 Ryan DP, Schapiro RH, Warshaw AL. Villous tumors of the duodenum. Ann Surg 1986; 203: 301 - 306

20 Clary BM, Tyler DS, Dematos $P$ et al. Local ampullary resection with careful intraoperative frozen section evaluation for presumed benign ampullary neoplasms. Surgery 2000; 127: 628-633

21 Blackman E, Nash SV. Diagnosis of duodenal and ampullary epithelia neoplasms by endoscopic biopsy: a clinicopathologic and immunohistochemical study. Hum Pathol 1985; 16: 901 -910

22 Menzel J, Poremba C, Dietl KH et al. Tumors of the papilla of Vater-inadequate diagnostic impact of endoscopic forceps biopsies taken prior to and following sphincterotomy. Ann Oncol 1999; 10: 1227-1231

23 Kiesslich R, Mergener K, Naumann C et al. Value of chromoendoscopy and magnification endoscopy in the evaluation of duodenal abnormalities: a prospective, randomized comparison. Endoscopy 2003; 35 : $559-563$

24 Dekker E, Boparai KS, Poley JW et al. High resolution endoscopy and the additional value of chromoendoscopy in the evaluation of duodenal adenomatosis in patients with familial adenomatous polyposis. Endoscopy 2009; 41: 666-669

25 Shahid MW, Buchner A, Gomez V et al. Diagnostic accuracy of probebased confocal laser endomicroscopy and narrow band imaging in detection of dysplasia in duodenal polyps. J Clin Gastroenterol 2012; 46: $382-389$

26 Hasama K. [An electron microscopic study on human intestinal epithelial cells during lipid absorption obtained by endoscopic biopsy under direct vision]. Nihon Shokakibyo Gakkai Zasshi 1982; 79: 1405-1411

27 Bulow S, Bjork J, Christensen IJ et al. Duodenal adenomatosis in familial adenomatous polyposis. Gut 2004; 53: 381 - 386

28 Groves CJ, Saunders BP, Spigelman AD et al. Duodenal cancer in patients with familial adenomatous polyposis (FAP): results of a 10 year prospective study. Gut 2002; 50: 636-641

29 Rex DK, Kahi C, O'Brien M et al. The American Society for Gastrointestinal Endoscopy PIVI (Preservation and Incorporation of Valuable Endoscopic Innovations) on real-time endoscopic assessment of the histology of diminutive colorectal polyps. Gastrointest Endosc 2011; 73: $419-422$

30 Maehata Y, Esaki M, Hirahashi M et al. Duodenal adenomatosis in Japanese patients with familial adenomatous polyposis. Dig Endosc 2014; 26: $30-34$ 